University of Nebraska - Lincoln

DigitalCommons@University of Nebraska - Lincoln

Architectural Engineering -- Faculty Publications

Architectural Engineering and Construction,

Durham School of

$4-2010$

\title{
Effects of Building Mechanical System Noise on Worker Performance and Perception
}

Lily M. Wang

University of Nebraska - Lincoln, Iwang4@unl.edu

Follow this and additional works at: https://digitalcommons.unl.edu/archengfacpub

Part of the Architectural Engineering Commons

Wang, Lily M., "Effects of Building Mechanical System Noise on Worker Performance and Perception" (2010). Architectural Engineering -- Faculty Publications. 6.

https://digitalcommons.unl.edu/archengfacpub/6

This Article is brought to you for free and open access by the Architectural Engineering and Construction, Durham School of at DigitalCommons@University of Nebraska - Lincoln. It has been accepted for inclusion in Architectural Engineering -- Faculty Publications by an authorized administrator of DigitalCommons@University of Nebraska Lincoln. 


\title{
Effects of building mechanical system noise on worker performance and perception ${ }^{\mathrm{a}}$
}

\author{
Lily M. Wang ${ }^{\mathrm{b}}$ \\ The Durham School of Architectural Engineering and Construction \\ University of Nebraska - Lincoln \\ 101A Peter Kiewit Institute \\ 1110 S. $67^{\text {th }}$ St. \\ Omaha, NE 68182-0681
}

This paper presents results from a number of studies that investigated the effects of noise from building mechanical systems on human task performance and perception. Three phases of research were conducted, each of which utilized a different set of noise signals produced by building mechanical systems: (1) broadband noise at different levels and spectral qualities; (2) tonal noise conditions; and (3) noise conditions with time-varying fluctuations. In each phase, at least six different noise signals (many based on in-situ measurements) were reproduced in an office-like setting. Thirty participants completed tasks (e.g. typing, grammatical reasoning, math) plus subjective questionnaires, while exposed to each noise condition for up to one hour. In general, no statistically significant differences were found in task performance across the various noise signals tested. However, higher annoyance/distraction responses from the test subjects were often significantly correlated with reduced typing performance. These higher annoyance/distraction responses were also closely correlated to higher subjective ratings of loudness, followed by roar, rumble, and tones or fluctuations. Of particular interest is that a greater perception of low frequency rumble was significantly linked to reduced performance on both the routine and cognitively demanding tasks. [Work supported by the American Society of Heating, Refrigeration and Air-Conditioning Engineers]

\section{INTRODUCTION}

Modern mechanical systems responsible for heating, ventilation and air-conditioning in buildings are sources of background noise that can cause occupant annoyance and reduced worker performance, particularly if there are prominent tonal components or time-varying fluctuations. Perceptible tones often result due to rotating parts, such as fans, motors, impellers, etc. Time-varying fluctuations may be due to ill-designed systems that demonstrate surging and excessive low frequency rumble, or may be on a larger timescale where the system's settings change over time, such as with variable air volume systems or systems switching on and off. The effects of such noise conditions on human performance and perception have not been systematically investigated, though, across a broad range of controlled conditions representing

\footnotetext{
${ }^{a}$ This proceedings paper presents a summary of results. For more detailed information, the reader is referred to the peer-reviewed publications to be published in ASHRAE Transactions, June 2010.

b Email address: LWang4@UNL.edu
} 
what can be found in existing spaces. This paper presents the results from a multi-phase study whose goal has been to determine how a variety of building mechanical system noise conditions, including those with varying degrees of tonal components or time-varying fluctuations, affect human performance and perception in a typical office setting.

Previous researchers have investigated effects of noise on human perception and performance, particularly at very high noise levels (higher than $70 \mathrm{dBA}$ ) and during the development of indoor noise rating methods like those presented in Ref. 1. One consistent finding is that while sound level is certainly an important factor, spectral characteristics of the noise also affect human perception and performance. In particular, noise with excessive low frequency energy or rumble has been shown in the lab and in the field to result in greater annoyance than equivalently loud signals without rumble. Noise conditions with rumble can also result in degradation in task performance, as shown by a number of researchers, although many of these studies compared only a few signals at a time. A detailed review of such previous work may be found in Refs. 2-4.

There have been some investigations, reviewed in Ref. 4, concerning effects on perception and performance of other random (or aperiodic) time-varying noise fluctuations, such as those that may occur on longer timescales than low frequency rumble (e.g. systems switching on and off) or involving different content (e.g. office noise including speech, equipment noise, etc.). Usually only one or two noise conditions were compared, with results indicating that random fluctuations can affect performance and annoyance.

The topic of noise with tones, particularly in terms of how the addition of tones impacts perception of loudness or annoyance, has also generated much interest over the years, as aircraft, industrial machinery, and other office equipment can generate such spectra. Ventilation-like noise spectra that specifically include tones have been utilized in a few investigations involving performance, and although not consistent, there has been some evidence that the presence of tones can cause degradation in performance, such as on figure identification tasks (see review in Ref. 3).

What differentiates the work reported herein from previous research is that a wider range of realistic spectra from building mechanical systems have been tested systematically, including ones with different tonal frequencies and tonal prominence ratios or featuring time-varying fluctuations, primarily in the low frequency region or on a larger timescale. Specifically, the effects of the noise on human task performance and perception in a typical office setting have been quantified using different types of tasks (typing, proofreading, grammatical reasoning and math tests) and subjective questionnaires.

\section{METHODOLOGY}

Three phases of research have been conducted, each of which utilized a different set of noise signals produced by building mechanical systems: (I) broadband noise at different levels and spectral qualities; (II) tonal noise conditions; and (III) noise conditions with time-varying fluctuations. In each phase, at least six different noise signals (many based on in-situ measurements) were reproduced in an office-like setting, and thirty participants completed tasks plus subjective questionnaires while exposed to each noise condition. All participants first underwent a series of pre-test screens to gauge that they met minimum required vision, hearing, and typing skills. Specifically regarding hearing, all test subjects demonstrated hearing thresholds below $25 \mathrm{~dB}$ hearing level in octave bands from $125 \mathrm{~Hz}$ to $8 \mathrm{kHz}$.

Testing was conducted in a $25.7 \mathrm{~m}^{3}$ indoor environmental test chamber at the University of Nebraska, outfitted as a typical office with two desks, carpet, gypsum board walls, and acoustical 
ceiling tile. The test chamber had an envelope with high sound transmission class of STC 47, low background noise level of RC 26(H), and a low reverberation time of 0.25 sec at $500 \mathrm{~Hz}$. During all tests, the chamber was thermally controlled to maintain a constant comfortable temperature of $20-22^{\circ} \mathrm{C}$. Overhead fluorescent lighting provided a constant average illuminance of 764 lux at the work plane. The sound in the test chamber was the only environmental characteristic that changed between test sessions, with the signals being presented in an inconspicuous manner over two loudspeakers: (i) an Armstrong i-ceiling loudspeaker which has the same appearance as the other ceiling tiles in the room, and (ii) a JBL Northridge E250P subwoofer, disguised to resemble an end table in the corner of the room.

For each phase, a repeated measures test design was used in which each subject was exposed to the same group of noise conditions. In Phase I, the exposure time was 15 minutes per noise signal with a 90 second adaptation period, while for the latter two phases, the exposure time was increased to 55 minutes each with the first 25 minutes being an adaptation period. The exposure time was increased based on results from an interim study ${ }^{5}$.

In Phase I, two types of tasks were tested on a computer: typing and proofreading. Each sequence of tasks was followed by a subjective questionnaire that asked the participant to rate his/her perception on discrete seven-point scales of the acoustic conditions in the space (loudness, rumble, roar, hiss, and annoyance), where 1 generally represented a low rating and 7 represented a high rating. In Phases II and III, more cognitively demanding tests were added, for a total of three: typing, grammatical reasoning, and math. The subjective questionnaire was also more extensive, including eight questions focused on perceptions related to the acoustic condition (loudness, rumble, roar, hiss, tones, changes over time, annoyance, and distraction) and five others focused on other conditions of the working environment (e.g. lighting, thermal comfort and indoor air quality). Since these other environmental conditions were kept constant and were not the focus of this investigation, the data are not presented further in this paper.

Multiple versions of all the tasks were utilized, and each participant completed the same versions of the tasks within each phase with the order of presentation randomized for each subject. The order in which each subject experienced the noise conditions was also randomized. Prior to testing, the subjects completed a PowerPoint tutorial on a computer that described the test procedures and introduced them to definitions and aural examples of the subjective terms if used in that phase: "rumbly", "roaring”, "hissy", and "tonal”. No other training was provided concerning the remaining descriptors on the questionnaire.

Further details on the methodology for each phase may be found in Refs. 2-4, respectively.

\subsection{Noise Conditions}

Table 1 lists the noise conditions used in each of the three phases of research along with their corresponding indoor noise ratings, based on measurements at the typical location of a participant's head. The loudness in sones was calculated per ANSI Standard S3.4 ${ }^{6}$. Procedures for calculating the other criteria are described in Ref. 1. For Phase I, the noise conditions (labeled I.1 to I.12) primarily varied in level and spectral quality. For Phase II, the noise conditions (labeled II.1 to II.6) had varying degrees of tonal components, many of which were based on in-situ recordings made in existing spaces that exhibited tones from mechanical systems. Prominence Ratio $(\mathrm{PR})^{7}$ has been used as the quantifier of tonalness in this investigation. It is a ratio of the power of the critical band centered on a tone compared to the mean power of the two adjacent critical bands, so it quantifies the degree of tonalness based on relative loudness differences, rather than based on whether the tone is masked within its critical 
band as the Tone-to-Noise Ratio ${ }^{7}$ does. To minimize the effect that different signal levels would have on human performance and perception and attempt to isolate any effect due to different degrees of tonalness, the sound levels of these six signals were adjusted so that they each produced a LAeq of approximately $47 \mathrm{dBA}$ in the test chamber.

For Phase III, the noise conditions (labeled III.1 to III.6) had varying degree of time-varying fluctuations, some of which were based on in-situ recording made in existing spaces that exhibited rumble or time-varying fluctuations from mechanical systems. Signal III.5 in particular was recorded in a university dormitory room, with a heat pump that was turned on and off at a rate of once every 30 seconds. Table 2 lists the six noise conditions used in Phase III along with metrics that have been suggested for quantifying the degree of low frequency fluctuations: Room Noise Criteria (RNC) ${ }^{8}$, and L1 - L99 [LF ave] ${ }^{9}$, where L1 is the level exceeded 1\% of the time and L99 is the level exceeded 99\% of the time. The L1 - L99 [LF ave] values were calculated by taking an energy average of the L1 - L99 quantities across the low frequency one-third octave bands from $12.5 \mathrm{~Hz}$ to $160 \mathrm{~Hz}$. The difference between the Cweighted equivalent sound pressure level and the A-weighted equivalent sound pressure level, LCeq - LAeq ${ }^{10}$, is also included in Table 2 as a measure of low frequency content. All three of these metrics seem to give roughly similar rankings to the signals regarding their low frequency fluctuation or content, with signals III.2 and III.6 having the highest values and signal III.5 having the lowest.

More detailed information on the noise signals used in each phase including their spectra may be found in Refs. 2-4, respectively.

\section{RESULTS}

Various statistical analysis methods in the software SPSS have been used to evaluate the results, typically with the independent variables being the different noise conditions tested in each phase and the dependent variables being the resulting task performance scores and subjective ratings for the questions regarding acoustics. Pearson's Product Moment Correlations and repeated measures analysis of variance (ANOVA) with Bonferroni post hoc tests have been applied to studying the task performance scores and the subjective questionnaire responses separately. Then these two groups of dependent variables have been related through a further statistical method, linear mixed models, to investigate significant correlations between performance and perception. For more details on the statistical methods used, see Ref. 11.

\subsection{Task Performance Results}

Across all phases of the research, results from repeated measures analysis of variance (ANOVA) indicate that the noise conditions tested did not have a significant main effect on any task performance. Figure 1 shows sample results of the math task for each noise condition in Phase III, averaged across all subjects; standard error of the mean bars are shown. This plot shows that, although not found to be statistically significant, there does appear to be a trend that the signals with the most low frequency content (III.2 and III.6) and the one with the on/off fluctuation (III.5) resulted in lower math scores than the other conditions tested. The reasoning task results were similar, whereas the typing results did not indicate any apparent trends. The results suggest that a large degree of low frequency fluctuations or large time-scale fluctuations may impact tasks that are more cognitively difficult, but not more routine tasks like typing. 


\subsection{Subjective Perception Results}

The subjective questionnaires in Phases II and III were more extensive than in Phase I, so results from those two phases will be discussed in this section. (Phase I results generally support the same findings.) In Phases II and III, six of the subjective questionnaire items were linked to subjective ratings of the noise signal characteristics (loudness, rumble, roar, hiss, tones, and changes in time), while the remaining two were linked to subjective responses due to the noise (annoyance and distraction). Pearson Product Moment Correlation analyses were run among these dependent variables on subjective perception, and many of the variables were found to be significantly correlated at the $\mathrm{p}<0.01$ level or $\mathrm{p}<0.05$ level.

The two subjective responses on annoyance and distraction had the highest correlation coefficient of 0.86 in Phase II and 0.91 in Phase III. This indicates that when participants gave higher responses on annoyance, they commonly gave higher responses on distraction as well. The next highest correlations were found between the rating of loudness and both responses of annoyance (0.77 in Phase II, 0.77 in Phase III) and distraction (0.71 in Phase II, 0.74 in Phase III). Of the noise characteristics evaluated, loudness perception is clearly the most highly linked to annoyance and distraction responses.

The noise characteristic ratings that were next highly correlated to annoyance in terms of correlation coefficients were roar (0.54), tones (0.50), and rumble ratings (0.48) in Phase II; results were similar for Phase III: roar (0.42), rumble (0.41), and changes in time ratings (0.40). These results confirm that loudness perception is often the noise characteristic most significantly linked to annoyance/distraction, but also show that the next characteristics connected to annoyance/distraction perception are the perceived amount of roar, rumble, and tones or timevarying fluctuations in the noise.

To determine if there were any significant effects of noise condition on the questionnaire responses, repeated measures ANOVA analyses were conducted. In both Phases II and III, results show that there was a main effect of noise condition on loudness ratings $(\mathrm{F}=3.40, \mathrm{p}<0.05$ for Phase II; $\mathrm{F}=8.83, \mathrm{p}<0.01$ for Phase III); participants did give different ratings on loudness perception between the six signals within each phase. Bonferroni post hoc tests were run to highlight statistically significant differences between the noise conditions in each phase, and show that perceptual ratings match well with the actual sone values for each signal, except for signal III.5. In Phase III, signal III.5 was rated to have the second highest loudness rating, but actually had the second lowest sone value. This appears to indicate that the larger timescale fluctuation from having the heat pump cycle on and off causes participants to rate the signal's perceived loudness to be higher than it is, even in its loudest mode.

ANOVA analysis also shows that there was a main effect of noise condition on tonal ratings in Phase II $(\mathrm{F}=4.84, \mathrm{p}<0.01)$; participants did perceive different degrees of tones in the six signals. Bonferroni post hoc tests show statistically significant differences as follows: (1) signal II.3 was rated to be significantly more tonal than II.4, and (2) signal II.5 was rated to be significantly more tonal than II.4 and II.1. Figure 2 plots these results and shows that, as might be expected, noise conditions with PR=9 (II.3, II.5, II.6) were generally perceived as more tonal than the other signals. However, signals II.3 and II.2 (both with $120 \mathrm{~Hz}$ tones but with different PR) were not found to be statistically different in tonal ratings, whereas II.5 and II.4 (both 235 $\mathrm{Hz}$ tones but with different PR) were; this lends further support to Hellweg Jr. and Nobile's results $^{12}$ that lower frequencies should require a higher $\mathrm{PR}$ to be considered prominent. The latest version of the ANSI S1.13 standard ${ }^{7}$ now reflects this.

For Phase III, the ANOVA analysis finds that there was a main effect of noise condition on the changes in time ratings $(\mathrm{F}=47.2, \mathrm{p}<0.01)$; that is, participants did perceive different degrees 
of time-varying fluctuations in the six signals (Figure 3). Bonferroni post hoc tests show that signal III.5 was rated to have significantly more changes in time than all other signals. Although not found to be statistically significant, Figure 3 also indicates a trend that noise conditions III.2 and III.6, which have the most low frequency fluctuations or content, were rated as demonstrating more changes in time than the other three signals.

The ANOVA results discussed in the previous three paragraphs confirmed that subjects perceived differences in loudness and tones or changes in time between the six test signals in Phase II or III. ANOVA main effects of noise condition on annoyance ( $\mathrm{F}=2.57, \mathrm{p}<0.05$ in Phase II; $F=10.9, p<0.01$ in Phase III) and distraction ( $F=2.32, p<0.05$ in Phase II; $F=10.6, p<0.01$ in Phase III) responses were also found, indicating that the participants did respond with different degrees of annoyance and distraction to the six noise conditions within each phase. In Phase II, the post hoc tests demonstrate that only signal II.1 was significantly found to be more annoying than signal II.4, but the trend as shown in Figure 4 is that signals with the higher PR of 9 in this study were perceived as more annoying and distracting than the ones with PR of 5.

In Phase III, the post hoc tests show that signals III.2 and III.5 were found to be significantly more annoying and distracting than all other signals (Figure 5). Signal III.2 has large low frequency content with an overall loudness of 11.4 sones, while III.5 has the large timescale fluctuation. The other signal with excessive low frequency energy (III.6), though, did not produce high ratings of annoyance and distraction. The author believes this indicates that loud signals with large amounts of low frequency energy correlate to greater annoyance/distraction responses, but quieter signals that have large amounts of low frequency fluctuation do not. This supports Kjellberg et al.'s ${ }^{13}$ suggestion that the overall level of the signal needs to be high enough so that excessive low frequency energy is perceptible.

\subsection{Relationships between Task Performance and Subjective Perception}

The testing in Phase I involved shorter noise exposure times and more routine tasks, rather than more cognitively demanding ones. Even so, a linear mixed model analysis shows significant relationships in this phase between lower typing performance and subjective perception of greater loudness $(F=5.72, p<0.05)$ or annoyance $(F=7.08, p<0.01)$.

In Phase II, the perception of tones was not significantly correlated to any task performance results, but there were other significant findings. Typing scores decreased as subjects perceived the noise to be more rumbly $(\mathrm{F}=13.52, \mathrm{p}<0.01)$, roaring $(\mathrm{F}=5.21, \mathrm{p}<0.05)$, or changing in time $(\mathrm{F}=3.81, \mathrm{p}<0.05)$ in character, and when they felt more annoyed $(\mathrm{F}=14.19, \mathrm{p}<0.01)$ or distracted $(\mathrm{F}=18.75, \mathrm{p}<0.01)$ by the sound. Figure 6(a) shows an example of the typing scores in relation to rumble ratings. (Note that the number above each standard error of the mean bar in Figure 6 indicates the number out of 360 observations that some participant assigned that rating.)

Math and reasoning task performances, however, actually improved with higher ratings of hiss or roar and only seemed to decrease somewhat with rumble ratings. The fact that a difference is found here between typing performance and math/reasoning performance is not unexpected, as the author and others have previously found that the type of task can affect results since different neural processes occur in accomplishing the tasks ${ }^{5}$; the typing task requires less cognitive thought than math/reasoning tasks. Low frequency rumble seems to be the only noise characteristic that generally produces lower scores for typing and math/reasoning performance, corroborating what has been found by other researchers ${ }^{14}$. With other noise characteristics such as roar and hiss, it could be that subjects feel annoyed or distracted so performance on a routine task like typing degrades, but the increased annoyance or distraction may compel subjects to 
focus more when working on cognitive tasks like math/reasoning, resulting in better performance scores.

Phase III results did not show any significant decrease in typing scores, unlike the previous two phases. Math and reasoning task performances did appear to decrease, though, with greater rumble ratings, as found in Phase II. Figure 6(b) shows the average reasoning scores decreasing with higher rumble ratings $(\mathrm{F}=4.81, \mathrm{p}<0.05)$; the average math scores also seemed to drop, although this was not found to occur at a statistically significant level.

There was furthermore no statistically significant decrease in task performance correlated to the changes in time ratings. Instead, a possible increase in math task performance was found $(\mathrm{F}=6.53, \mathrm{p}<0.05)$. This suggests that participants may have been increasingly aroused by the higher degrees of changes over time in the noise used in this study and subsequently focused and performed better on the task at hand, in accordance with arousal theory. However, as other research has indicated, being exposed to even longer periods of fluctuating noise than the one hour used in the current investigation may have further detrimental effects, including increased feelings of fatigue and stress ${ }^{15}$.

\section{CONCLUSIONS}

The results of this multi-phase study can help to answer two central questions, regarding noise characteristics of building mechanical systems, subjective perception, and task performance. The first is: which noise characteristics are linked to higher annoyance and distraction responses? Among the noise characteristics surveyed, it was found that loudness perception is most closely linked to annoyance/distraction, followed by the perception of roar, rumble, and tones or time-varying fluctuations in the noise. Consequently, in designing commercial office buildings, the degree of loudness, roar, rumble, and tones or time-varying fluctuations in the background noise should be minimized to optimize worker comfort. Particularly with regards to tones, certain signals with tones of $P R=9$ were generally perceived in this project as more annoying than those of $\mathrm{PR}=5$, but more research should be conducted to investigate a wider range of tonal prominence ratios across different frequencies. With regards to changes in time, signals louder than $50 \mathrm{dBA}$ with excessive low frequency content and signals with larger timescale variations, such as the heat pump cycling on and off every 30 seconds, were generally perceived in this project as more annoying that the others tested, but more research is also suggested in this arena to quantify further guidelines for time-varying fluctuations in order to minimize their negative effects on human perception and task performance.

The second question is: which noise characteristics are linked to lower task performance scores? The study did find that signals perceived to be more rumbly generally produced lower performance on typing and math/reasoning tasks. This result further supports the fact that the degree of low frequency rumble should be minimized in background noise conditions of offices, not only for occupant comfort but also for improved performance. Furthermore, statistically significant relationships were found between higher annoyance/distraction responses and lower typing performance, so reducing occupant annoyance/distraction by reducing the other perceived characteristics of loudness, roar, and tones or time-varying fluctuations, may have additional benefits on performance. 


\section{ACKNOWLEDGEMENTS}

This work has been funded by the American Society of Heating, Refrigeration, and AirConditioning Engineers (ASHRAE). The author would like to thank the many students who worked as research assistants on the project, including Erica Ryherd, Cathleen Novak, Marc Choiniere, Megan Christensen, and Jennifer Reisdorff.

\section{REFERENCES}

1. ASHRAE, "Sound and vibration control", Chap. 47 in ASHRAE Handbook-HVAC Applications, American Society of Heating, Refrigerating and Air-Conditioning Engineers, Inc, Atlanta, (2007).

2. E. E. Bowden and L. M. Wang, "Relating human productivity and annoyance to indoor noise criteria systems: a low frequency analysis”, ASHRAE Transactions, 111(1), 684-692, (2005).

3. E. E. Ryherd and L. M. Wang, "The effects of noise from building mechanical systems with tonal components on human performance and perception (1322-RP)", accepted for publication in ASHRAE Transactions, (2010).

4. L. M. Wang and C. C. Novak, "Human performance and perception-based evaluations of indoor noise criteria for rating mechanical system noise with time-varying fluctuations (1322RP)", in revision for publication in ASHRAE Transactions, (2010).

5. E. E. Ryherd and L. M. Wang, "Effects of exposure duration and type of task on subjective performance and perception in noise”, Noise Control Eng. J., 55(3), 334-347, (2007).

6. ANSI, ANSI S3.4: Procedure for the Computation of Loudness of Steady Sounds, Acoustical Society of America, Melville, NY, (2005).

7. ANSI, ANSI S1.13: Measurement of Sound Pressure Levels in Air, Acoustical Society of America, Melville, NY, (2005).

8. ANSI, ANSI S12.2: Criteria for Evaluating Room Noise, Acoustical Society of America, Melville, NY, (2008).

9. J. A. Mann III, J. Kading, and M. B. Pate, RP-1219 Final Report: Duct Rumble Noise Resulting from Aerodynamic System Effects at the Discharge of a Centrifugal Fan, American Society of Heating, Refrigeration and Air-Conditioning Engineers, Atlanta, (2007).

10. N. Broner and H. G. Leventhall, "Low frequency noise annoyance assessment by Low Frequency Noise Rating (LFNR) curves”, J. Low Freq. Noise Vib., 2, 20-28, (1983).

11. A. Field and G. Hole, How to Design and Report Experiments, Sage Publications, London, (2003).

12. R. D. Hellweg Jr. and M. A. Nobile, "Modification to procedures for determining prominent discrete tones", Proceedings of Internoise, 111(1), 1707-1714, (2002). 
13. A. Kjellberg, M. Tesarz, K. Holmberg, and U. Landström, "Evaluation of frequencyweighted sound level measurements for prediction of low-frequency noise annoyance”, Environment International, 23, 519-527, (1997).

14. G. Leventhall, P. Pelmear, and S. Benton, A Review of Published Research on Low Frequency Noise and Its Effects, Defra Publications, London, (2003).

15. T. Witterseh, D. P. Wyon, and G. Clausen, "The effects of moderate heat stress and openplan office noise distraction on SBS symptoms and the performance of office work", Indoor Air, 14(8), 30-40, (2004).

Table 1 - Noise conditions used in each phase and their corresponding indoor noise criteria ratings, with $N=$ neutral, $R=$ rumbly, $H=$ hissy, $V=$ vibrational, $L F=$ excessive low frequency, $M F=$ excessive mid frequency (roaring in character), and HF = excessive high frequency.

\begin{tabular}{|c|c|c|c|c|c|c|}
\hline $\begin{array}{c}\text { Noise Condition Label and } \\
\text { Description }\end{array}$ & NC & NCB & RC & RC-Mark II & $\begin{array}{c}\mathbf{L}_{\text {Aeq }} \\
(\text { dBA }) \\
\end{array}$ & $\begin{array}{c}\text { Loudness } \\
\text { (sones) }\end{array}$ \\
\hline \multicolumn{7}{|c|}{ PHASE I } \\
\hline I.1 Low-level neutral & 30 & $29(\mathrm{RH})$ & $31(\mathrm{H})$ & $\begin{array}{l}31(\mathrm{LF}), \\
\text { marginal }\end{array}$ & 38 & - \\
\hline I.2 Low-level rumbly & 31 & $30(\mathrm{RH})$ & $32(\mathrm{RH})$ & $\begin{array}{c}32(\mathrm{~N}), \\
\text { acceptable }\end{array}$ & 39 & - \\
\hline I.3 Low-level roaring & 41 & $33(\mathrm{R})$ & $35(\mathrm{R})$ & $\begin{array}{l}35(\mathrm{MF}), \\
\text { marginal }\end{array}$ & 46 & - \\
\hline I.4 Low-level hissy & 39 & $34(\mathrm{H})$ & $35(\mathrm{H})$ & $\begin{array}{c}35 \text { (HF), } \\
\text { objectionable }\end{array}$ & 42 & - \\
\hline I.5 Mid-level neutral & 40 & 37 (R) & $40(N)$ & $\begin{array}{l}40(\mathrm{HF}), \\
\text { marginal }\end{array}$ & 47 & - \\
\hline I.6 Mid-level rumbly & 44 & $37(\mathrm{R})$ & $40(\mathrm{R})$ & $\begin{array}{c}40(\mathrm{~N}), \\
\text { acceptable }\end{array}$ & 48 & - \\
\hline I.7 Mid-level roaring & 53 & $40(\mathrm{R})$ & $43(\mathrm{R})$ & $\begin{array}{c}43(\mathrm{MF}), \\
\text { objectionable }\end{array}$ & 55 & - \\
\hline I.8 Mid-level hissy & 46 & $42(\mathrm{H})$ & $43(\mathrm{H})$ & $\begin{array}{c}43 \text { (HF), } \\
\text { objectionable }\end{array}$ & 50 & - \\
\hline I.9 High-level neutral & 53 & $47(\mathrm{R})$ & $49(\mathrm{~N})$ & $\begin{array}{l}49(\mathrm{MF}), \\
\text { marginal }\end{array}$ & 57 & - \\
\hline I.10 High-level rumbly & 59 & $47(\mathrm{R})$ & $50(\mathrm{RV})$ & $\begin{array}{l}50(\mathrm{~V}), \\
\text { marginal }\end{array}$ & 58 & - \\
\hline I.11 High-level roaring & 64 & $49(\mathrm{R})$ & $53(\mathrm{R})$ & $\begin{array}{c}53(\mathrm{MF}), \\
\text { objectionable }\end{array}$ & 66 & - \\
\hline I.12 High-level hissy & 55 & $52(\mathrm{H})$ & $53(\mathrm{H})$ & $\begin{array}{c}53(\mathrm{HF}), \\
\text { objectionable }\end{array}$ & 59 & - \\
\hline \multicolumn{7}{|c|}{ PHASE II } \\
\hline II.1: Mid-level neutral & 40 & $38(\mathrm{~N})$ & $40(\mathrm{~N})$ & $\begin{array}{l}40(\mathrm{HF}), \\
\text { marginal }\end{array}$ & 47 & 8.8 \\
\hline $\begin{array}{c}\text { II.2: } 120 \mathrm{~Hz} \text { tonal noise } \\
\text { PR }=5\end{array}$ & 40 & $38(\mathrm{~N})$ & $41(\mathrm{~N})$ & $\begin{array}{l}41(\mathrm{HF}), \\
\text { marginal }\end{array}$ & 47 & 7.5 \\
\hline $\begin{array}{c}\text { II.3: } 120 \mathrm{~Hz} \text { tonal noise } \\
\text { PR = } 9 \\
\end{array}$ & 44 & 38 (R) & $41(\mathrm{~N})$ & $\begin{array}{l}41(\mathrm{HF}), \\
\text { marginal }\end{array}$ & 48 & 7.5 \\
\hline $\begin{array}{c}\text { II.4: } 235 \mathrm{~Hz} \text { tonal noise } \\
\text { PR = } 5 \\
\end{array}$ & 41 & $37(\mathrm{H})$ & $40(\mathrm{~N})$ & $\begin{array}{c}40 \text { (HF), } \\
\text { objectionable }\end{array}$ & 46 & 7.0 \\
\hline $\begin{array}{c}\text { II.5: } 235 \mathrm{~Hz} \text { tonal noise } \\
\text { PR }=9\end{array}$ & 42 & $37(\mathrm{R}, \mathrm{H})$ & $41(\mathrm{~N})$ & $\begin{array}{c}41(\mathrm{HF}), \\
\text { objectionable }\end{array}$ & 47 & 7.1 \\
\hline
\end{tabular}




\begin{tabular}{|c|c|c|c|c|c|c|}
\hline $\begin{array}{c}\text { II.6: } 595 \mathrm{~Hz} \text { tonal noise } \\
\text { PR }=9\end{array}$ & 43 & $37(\mathrm{R}, \mathrm{H})$ & $39(\mathrm{H})$ & $\begin{array}{c}39(\mathrm{~N}), \\
\text { acceptable }\end{array}$ & 47 & 8.2 \\
\hline \multicolumn{7}{|c|}{ PHASE III } \\
\hline III.1: Mid-level neutral & 45 & $41(\mathrm{RV})$ & $44(\mathrm{~V})$ & $\begin{array}{c}44(\mathrm{~V}), \\
\text { marginal }\end{array}$ & 51 & 10.7 \\
\hline III.2: Mid-level rumbly & 56 & $41(\mathrm{RV})$ & $44(\mathrm{RV})$ & $\begin{array}{c}44(\mathrm{LF}, \mathrm{V}), \\
\text { marginal }\end{array}$ & 53 & 11.4 \\
\hline $\begin{array}{l}\text { III.3: Duct rumble from centrifugal fan } \\
\text { (35\% fan load curve, } 2.5 \% \text { sp) }\end{array}$ & 42 & $38(\mathrm{RV})$ & $41(\mathrm{~V})$ & $\begin{array}{l}41(\mathrm{~V}), \\
\text { marginal }\end{array}$ & 48 & 9.1 \\
\hline $\begin{array}{l}\text { III.4: Duct rumble from centrifugal fan } \\
\text { (80\% fan load curve, } 1 \text { ” sp) }\end{array}$ & 42 & $38(\mathrm{R})$ & $41(\mathrm{~N})$ & $\begin{array}{c}41(\mathrm{~N}), \\
\text { acceptable }\end{array}$ & 48 & 8.5 \\
\hline $\begin{array}{l}\text { III.5: Heat pump recording, } \\
\text { cycling on and off }\end{array}$ & 39 & $37(\mathrm{H})$ & $38(\mathrm{~N})$ & $\begin{array}{c}38(\mathrm{~N}), \\
\text { acceptable }\end{array}$ & 44 & 7.2 \\
\hline $\begin{array}{c}\text { III.6: Low-level } \\
\text { low frequency fluctuating }\end{array}$ & 46 & $29(\mathrm{RV})$ & 30 (RHV) & $\begin{array}{c}30(\mathrm{LF}, \mathrm{V}) \\
\text { objectionable }\end{array}$ & 45 & 5.3 \\
\hline
\end{tabular}

Table 2 - Noise conditions used in Phase III and their corresponding quantifiers for degree of low frequency fluctuations or content.

\begin{tabular}{|c|c|c|c|}
\hline $\begin{array}{c}\text { Noise Condition Label and } \\
\text { Description }\end{array}$ & RNC & $\begin{array}{c}\text { L1 - L99 [LF ave] } \\
\text { (dB) }\end{array}$ & $\begin{array}{c}\mathbf{L}_{\text {Ceq }}-\mathbf{L}_{\text {Aeq }} \\
\text { (dB) }\end{array}$ \\
\hline III.1: Mid-level neutral & $45(250 \mathrm{~Hz})$ & 48.8 & 18.9 \\
\hline III.2: Mid-level rumbly & $56(63 \mathrm{~Hz})$ & 57.7 & 26.2 \\
\hline $\begin{array}{c}\text { III.3: Duct rumble from centrifugal fan } \\
\text { (35\% fan load curve, 2.5” sp) }\end{array}$ & $41(500 \mathrm{~Hz})$ & 44.3 & 16.9 \\
\hline $\begin{array}{c}\text { III.4: Duct rumble from centrifugal fan } \\
\text { (80\% fan load curve, 1” sp) }\end{array}$ & $42(125 \mathrm{~Hz})$ & 43.9 & 9.5 \\
\hline $\begin{array}{c}\text { III.5: Heat pump recording, } \\
\text { cycling on and off }\end{array}$ & $38(1000 \mathrm{~Hz})$ & 33.4 & 27.4 \\
\hline $\begin{array}{c}\text { III.6: Low-level } \\
\text { low frequency fluctuating }\end{array}$ & $60(31.5 \mathrm{~Hz})$ & 52.4 & \\
\hline
\end{tabular}

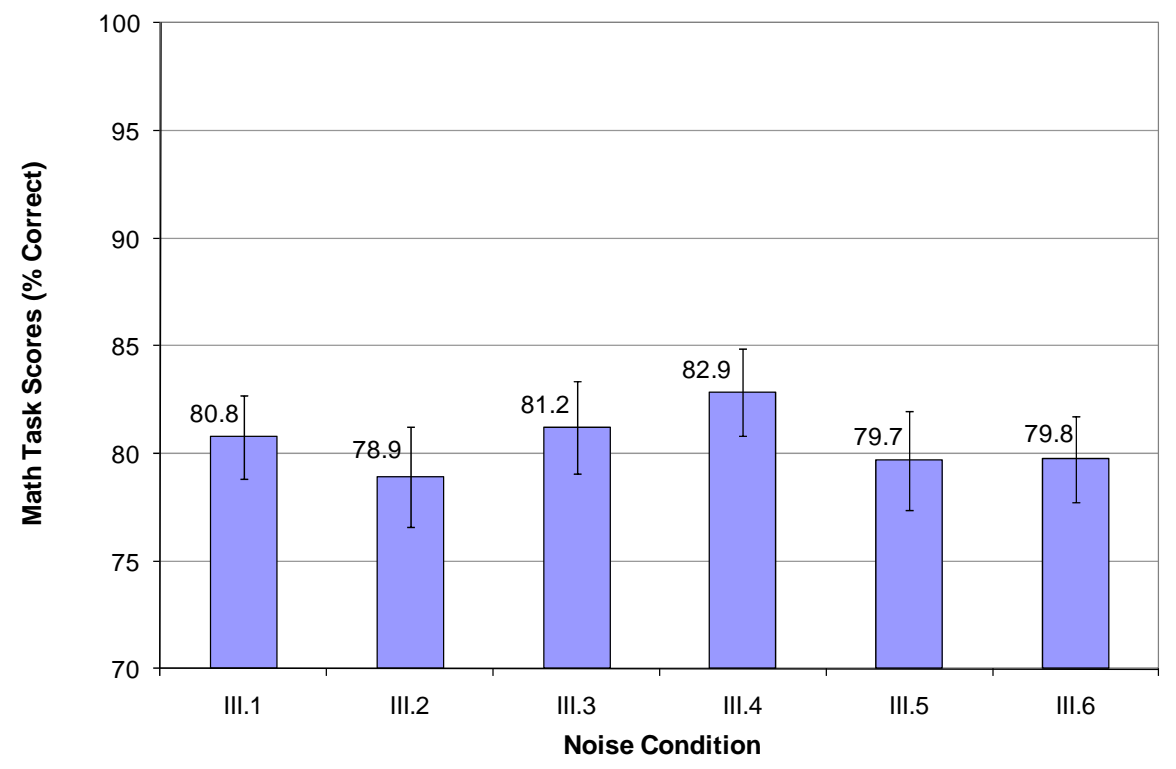

Fig. 1 - The math task performance scores in percent correct for each noise condition in Phase III, averaged across all subjects. The bars represent the standard error of the means. 


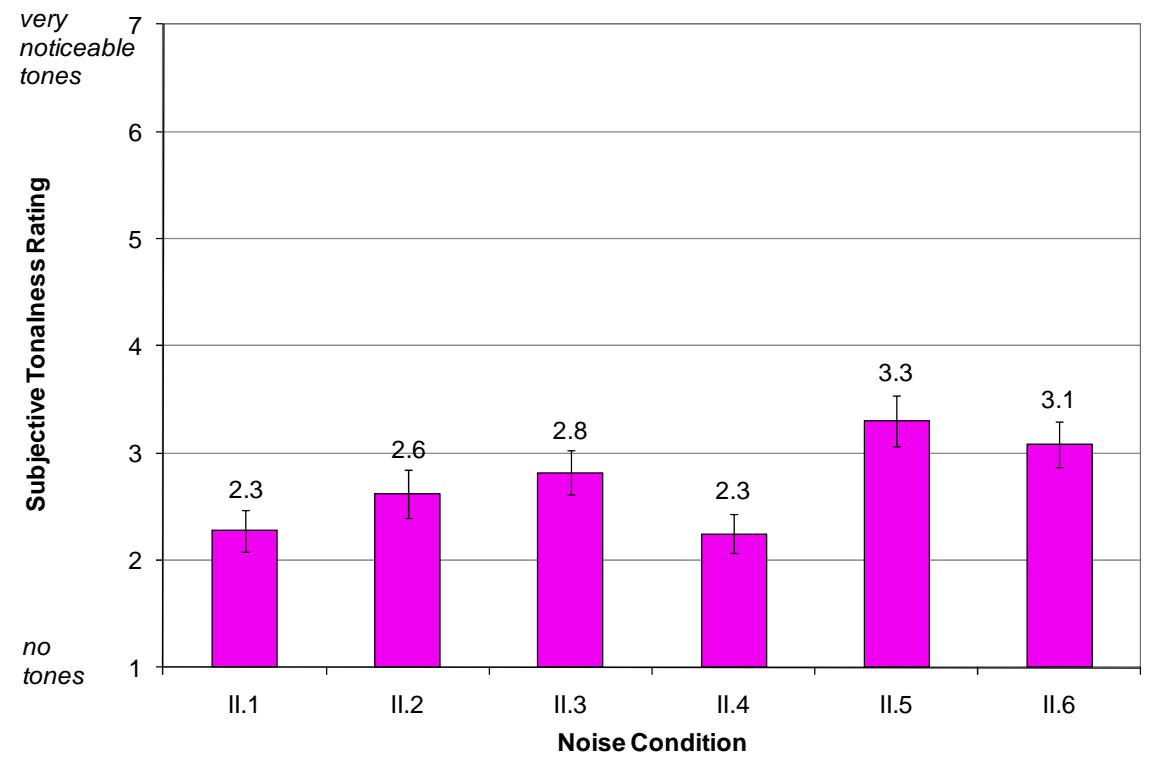

Fig. 2 - Subjective tonalness ratings of the various noise conditions in Phase II, averaged across all subjects. The bars represent the standard error of the means.

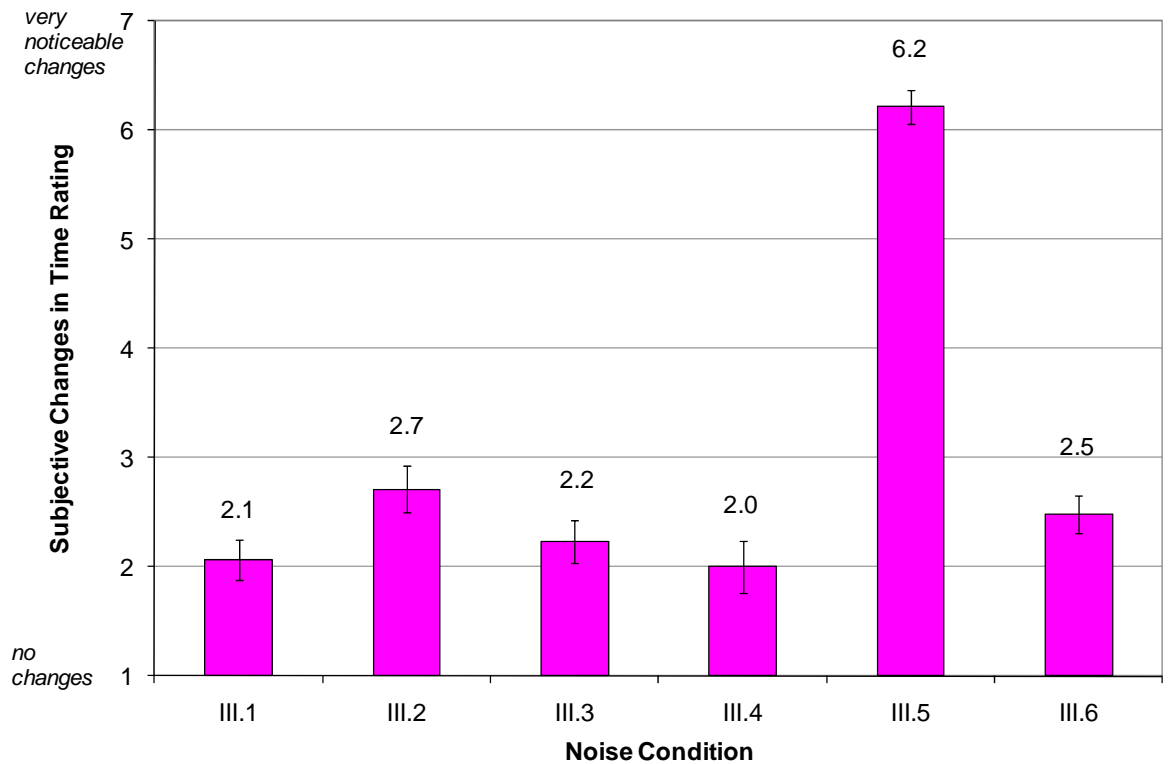

Fig. 3 - Subjective changes-in-time ratings of the various noise conditions in Phase III, averaged across all subjects. The bars represent the standard error of the means. 


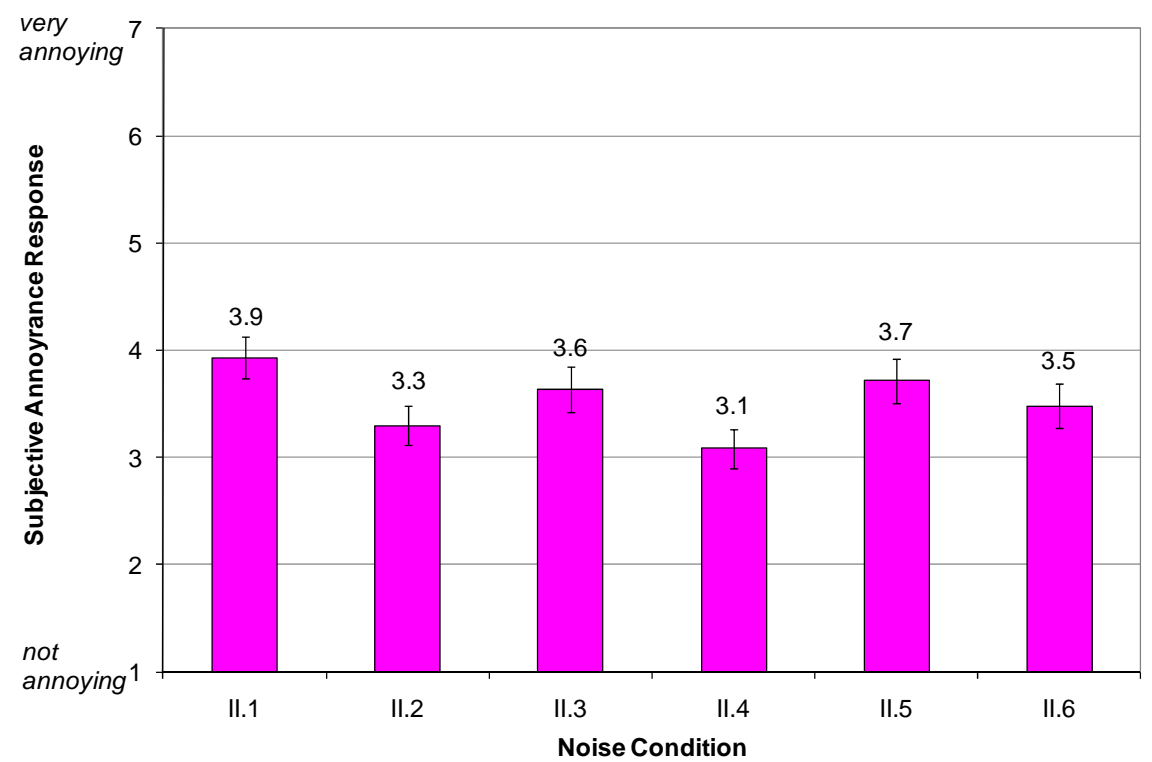

Fig. 4 - Subjective annoyance responses to the various noise conditions in Phase II, averaged across all subjects. The bars represent the standard error of the means.

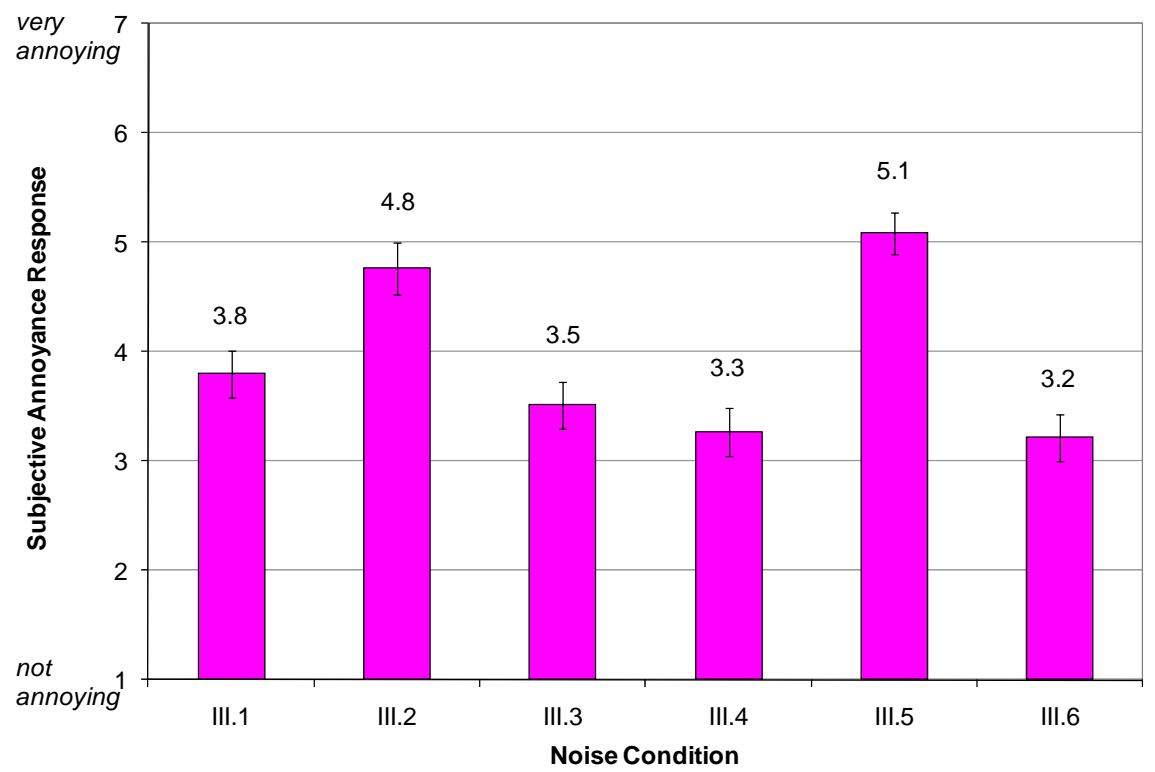

Fig. 5 - Subjective annoyance responses to the various noise conditions in Phase III, averaged across all subjects. The bars represent the standard error of the means. 


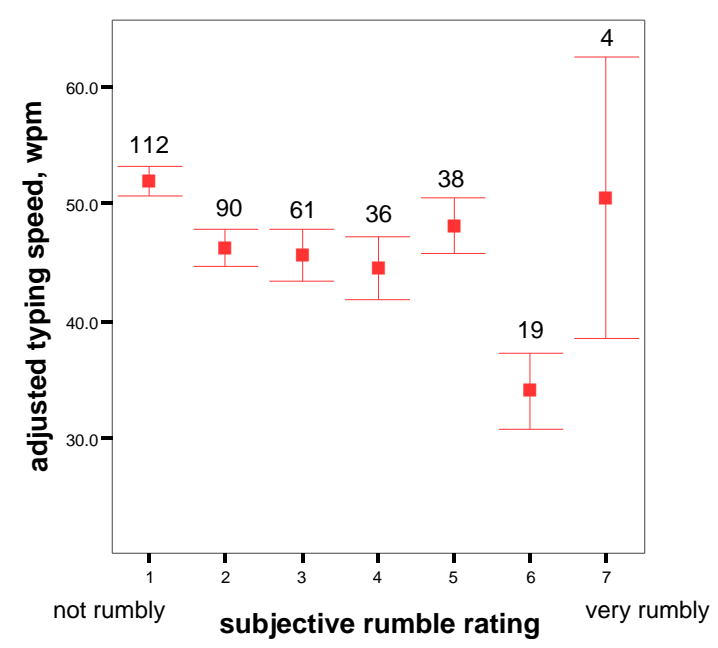

(a)

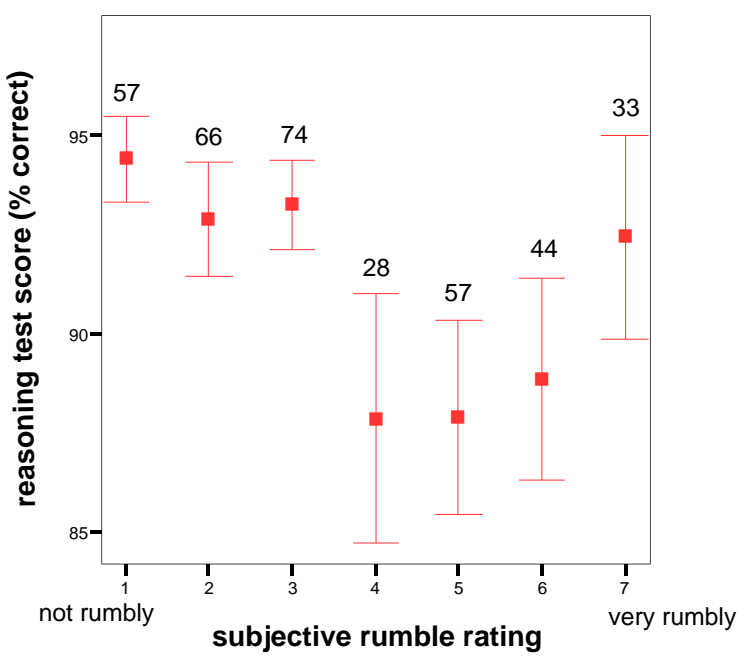

(b)

Fig. 6 - (a) The average adjusted typing speed in words per minute at each subjective rumble rating value from Phase II, and (b) the average reasoning score in percent correct at each subjective rumble rating value from Phase III. The bars represent standard error of the means. Numbers above the bars represent the number of observations out of 360 in which a participant gave this rating to a noise condition. 